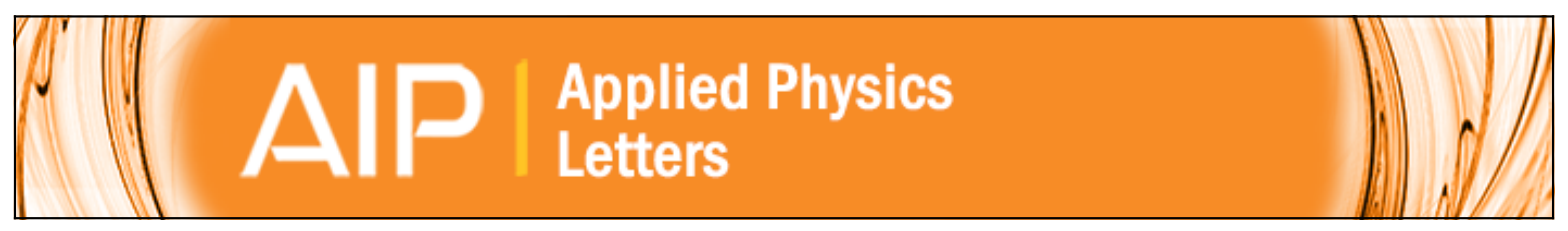

Evidence for population inversion in excited electron states of a double barrier resonant tunneling structure

J. W. Cockburn, M. S. Skolnick, D. M. Whittaker, P. D. Buckle, A. R. K. Willcox, and G. W. Smith

Citation: Applied Physics Letters 64, 2400 (1994); doi: 10.1063/1.111627

View online: http://dx.doi.org/10.1063/1.111627

View Table of Contents: http://scitation.aip.org/content/aip/journal/apl/64/18?ver=pdfcov

Published by the AIP Publishing

AlP Re-register for Table of Content Alerts

Create a profile. 


\title{
Evidence for population inversion in excited electron states of a double barrier resonant tunneling structure
}

\author{
J. W. Cockburn, M. S. Skolnick, D. M. Whittaker, P. D. Buckle, and A. R. K. Willcox \\ Department of Physiss, Iniversity of Sheffeld, Sheffield S3 7RH, United Kingdom \\ G. W. Smith \\ Defence Research Agency, St. Andrew's Road, Malvern, WR14 3PS, United Kingdom
}

(Received 16 November 1993; accepted for publication 9 February 1994)

\begin{abstract}
We report evidence for a population inversion between excited electron states of the quantum well of a GaAs-AIGaAs double barrier resonant tunneling structure (DBRTS). The relative populations of the states are determined by photoluminescence spectroscopy of the tunneling electrons in the structure. When the DBRTS is biased at the fourth electron resonance, the population of the $n=4$ confined level is found to be greater than that of the $n=3$ state. We show that such a population inversion is consistent with a rate equation analysis of the relative populations of the two levels when electrons tunnel into $n=4$.
\end{abstract}

During the last 20 years, there have been a variety of proposals to achieve a population inversion between electron energy levels in semiconductor low dimensional structures, based on the ability to manipulate independently the populations of individual levels in such structures. ${ }^{1-7}$ For example, variation of quantum well (QW) width permits modification of interlevel scattering rates, whereas rates of filling and emptying of the levels by tunneling can be altered by changing barrier widths. However, in spite of this effort, a report of population inversion in such a device structure is lacking up to the present time. In this letter we report the results of photoluminescence (PL) experiments which provide evidence for a population inversion between the $n=4$ (E4) and $n=3$ (E3) levels of the $200-\AA$-wide $\mathrm{QW}$ of a GaAs-AIGaAs double barrier resonant tunneling structure (DBRTS). We show that such a population inversion is consistent with a rate analysis of the excited state populations, since the electron-optic phonon scattering rate is greater for E3-E2 transitions than for E4-E3 transitions.

The DBRTS was grown by molecular beam epitaxy and comprised: $n^{+}$GaAs substrate, $0.5 \mu \mathrm{nn} n=1.5 \times 10^{18} \mathrm{~cm}^{-3}$ GaAs, $0.5 \mu \mathrm{m} 2 \times 10^{17} \mathrm{~cm}^{-3}$ GaAs, $100 \AA$ undoped GaAs, $85 \AA$ undoped $\mathrm{Al}_{6.33} \mathrm{Ga}_{0.67} \mathrm{As}$ barrier, $200 \AA$ undoped $\mathrm{GaAs}$ QW, $85 \AA$ undoped $\mathrm{Al}_{0.33} \mathrm{Ga}_{0.67} \mathrm{As}$ barrier, $100 \AA$ undoped GaAs, $0.75 \mu \mathrm{m} n=2 \times 10^{17} \mathrm{~cm}^{-3} \mathrm{GaAs}, 0.25 \mu \mathrm{m} n=1 \times 10^{18}$ $\mathrm{cm}^{-3}$ GaAs top contact. The structure was processed into mesas with annular contacts. PL was excited by a He-Ne laser, using a power density of around $1 \mathrm{~W} \mathrm{~cm}^{-2}$ which produced negligible perturbation of the $I-V$ characteristics. The $I-V$ characteristic obtained at $T=5 \mathrm{~K}$ is shown in Fig. 1(a). Electron tunneling resonances are observed at $0.05,0.18$, 0.40 , and $0.68 \mathrm{~V}$.

When positive bias is applied, photocreated minority holes are driven from the top contact to the collector barrier. They then tunnel into the $\mathrm{QW}$, where they can recombine with electrons to generate PL. Throughout the bias range of the experiment the most intense PL from the $\mathrm{QW}$ arises from recombination of E1 clectrons with $n=1$ (IHH1) heavy holes $\left(E_{1 \mathrm{~h}}\right.$ recombination). At biases beyond the onset of the $E 2$ resonance, $\mathrm{E} 2-\mathrm{HH} 1\left(\mathrm{E}_{2 \mathrm{~h}}\right)$ recombination is expected to occur at $1.551 \mathrm{eV}$. For most of this bias range, the $E_{21 \mathrm{~h}} \mathrm{PL}$ is obscured by overlap with the high energy tail of the strong GaAs band-edge PL. However, at the peak of the E2 resonance $(\sim 0.17 \mathrm{~V})$ a weak shoulder at $1.554 \mathrm{eV}$ becomes discernible on the GaAs high energy tail, due to $E_{2 \mathrm{~h}}$ recombination.

At a bias of around $0.25 \mathrm{~V}$, close to the onset of the $\mathrm{E} 3$ resonance, an additional PL peak emerges at an energy of $1.596 \mathrm{eV}$. This peak, which is about $10^{4}$ times less intense than the $\mathrm{E}_{1 \mathrm{lh}}$ peak, is attributed to $\mathrm{E} 3-\mathrm{HH} 1\left(\mathrm{E}_{3 \mathrm{~h}}\right)$ recombina-

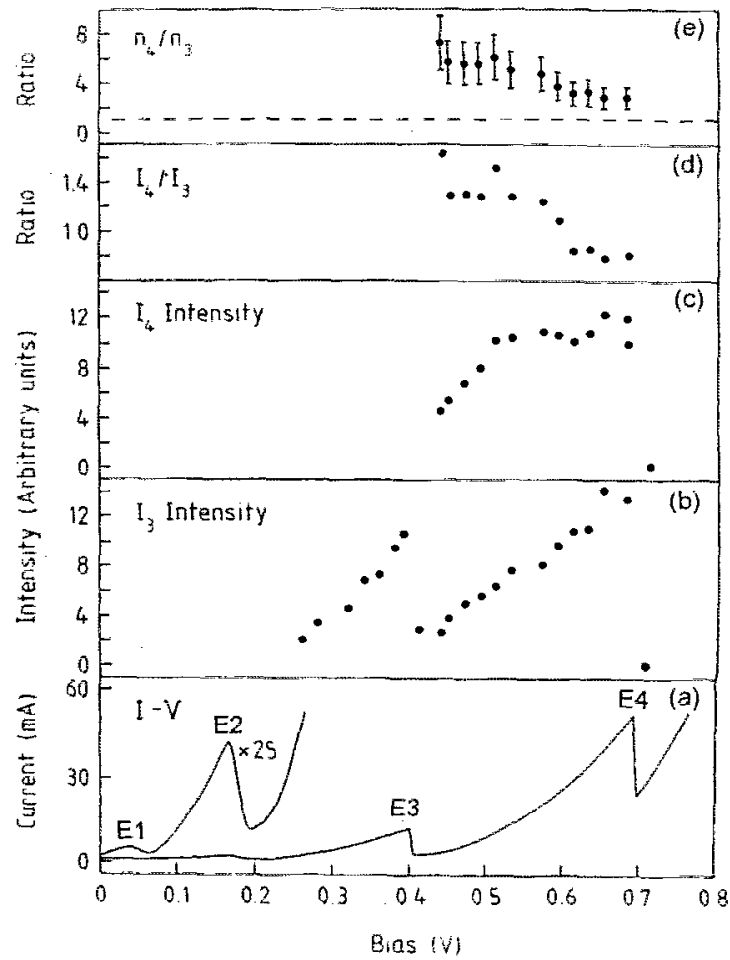

FIG, 1. (a) Current-voltage characteristic (5 K) showing E1, E2, E3, and E4 electron tunneling resonances. (b) E3-HH1 PL intensity $\left(I_{3}\right)$ vs bias in the bias range of the $\mathrm{E} 3$ and $\mathrm{E} 4$ resonances. (c) E4-HHI PI. intensity $\left(I_{4}\right)$ vs bias in the bias range of the $\mathrm{E} 4$ resonance. (d) $l_{4} / I_{3}$ vs bias. (e) $n_{4} / n_{3}$ population ratio is bias. 


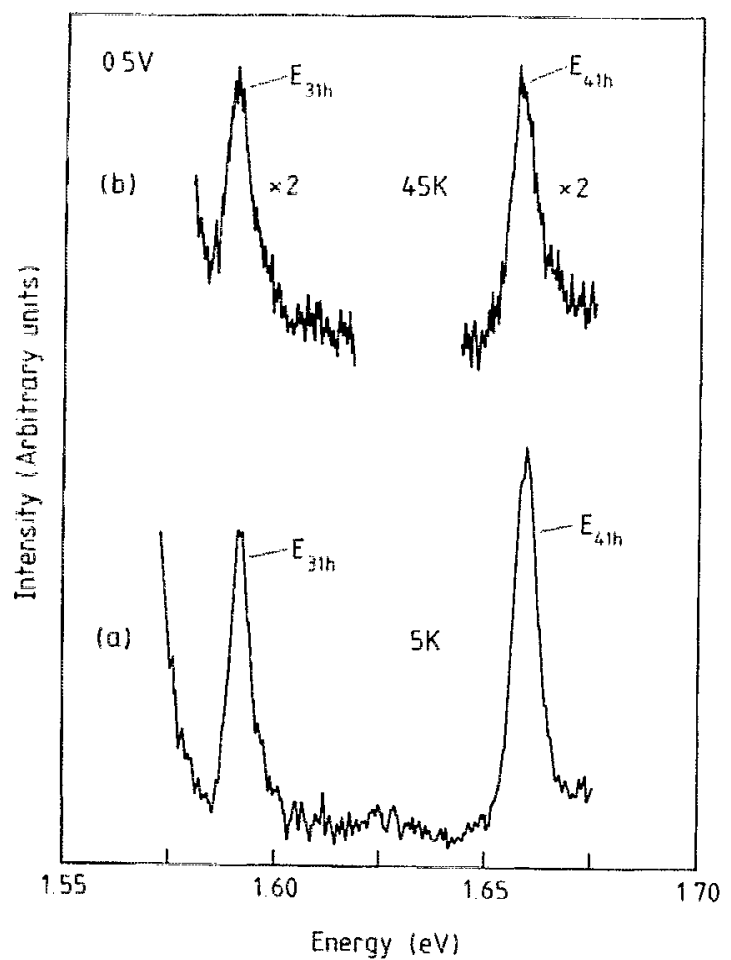

FIG. 2. (a) PL spectrum obtained at a bias of $0.5 \mathrm{~V}$ and a temperature of 5 $\mathrm{K}$ showing $E_{3 \mathrm{~h}}$ and $\mathrm{E}_{4 \mathrm{~h}}$ recombination. (b) As (a), but at $T=45 \mathrm{~K}$.

tion, calculated to occur at $1.593 \mathrm{eV}$. The integrated intensity $\left(I_{3}\right)$ of this peak is plotted versus bias in Fig. 1(b). At the onset of the $\mathrm{E} 4$ resonance $(V \sim 0.44 \mathrm{~V})$, a further PL peak is observed at $1.660 \mathrm{eV}$, due to $\mathrm{E} 4-\mathrm{HH} 1\left(E_{4 \mathrm{~h}}\right)$ recombination (calculated to occur at $1.650 \mathrm{eV}$ ). The integrated intensity of the $E_{\text {flh }}$ peak is plotted against bias in Fig. 1(c).

A representative $\mathrm{PL}$ spectrum in the $1.55-1.70 \mathrm{eV}$ region, obtained at a bias of $0.5 \mathrm{~V}$, is shown in Fig. 2(a). The $I_{4} / l_{3}$ ratio, which is subject to an experimental uncertainty of $\pm 1.5 \%$, is plotted versus bias in Fig. 1(d). The population ratio of $\mathrm{E} 4$ and $\mathrm{E} 3, n_{4} / n_{3}$, is determined from $I_{4} / I_{3}$ using the relation

$$
(\alpha / \beta)\left(I_{4} / I_{3}\right)=\left(n_{4} / n_{3}\right)\left(f_{4} / f_{3}\right),
$$

where $\alpha(=1.2 \pm 0.1)^{5}$ accounts for the greater absorption of $E_{4 i h}$ than $E_{3 h h} P L$ in the GaAs top contact, $\beta(-1.6 \pm 0.1)$ is the correction factor for the response of the spectrometer/ detector system and $f_{4} / f_{3}$ is the ratio of the oscillator strengths for $E_{4 h \mathrm{~h}}$ and $E_{31 \mathrm{~h}}$ transitions at the electric field of interest.

Equation (1) assumes that the entire electron distributions in both $\mathrm{E} 3$ and $\mathrm{E} 4$ are probed by $\mathrm{HH} 1$ holes. Assuming conservation of transverse wave vector, $k_{x y}$, this means that the maximum $k_{x y}$ of the holes must be at least as large as the maxinum $k_{x y}$ of electrons in E3 and E4 if Eq. (1) is to be valid. We estimate a $\mathrm{HH} 1$ hole temperature of around $10 \mathrm{~K}$, based on published cooling rates for holes in GaAs $\mathrm{QWs}^{6}$ and a HH1 hole lifetime $\geqslant 10 \mathrm{~ns}^{7}$ Using the Luttinger Hamiltonian to calculate the HH1 dispersion relation for our potential, we obtain a maximum $k_{x y}$ of $-7.5 \times 10^{5} \mathrm{~cm}^{-1}$ for HH1 holes at $10 \mathrm{~K}$. For an electron effective mass of 0.067 $m_{11}$, the above condition requires that E3 and E4 clectrons relax to within about $3 \mathrm{meV}$ of the bottom of their respective subbands. Any higher energy electrons may be detected in PL by raising the temperature of the sample in order to provide a thermal population of holes at increased $k_{x y}$. The presence of any high energy electron distributions would then lead to broadening of the $E_{31 h}$ and $E_{4 h \mathrm{~h}}$ PL peaks. If different E3 and E4 distributions were probed, this would result in changes in the $I_{4} / I_{3}$ ratio as the temperature was raised. In order to check the validity of Eq. (1) we therefore studied $\mathrm{PL}$ at higher temperatures. The $I_{4} / I_{3}$ ratio at $0.5 \mathrm{~V}$ remained at a value of $1.5 \pm 0.1$ as the sample temperature was raised from 5 to $45 \mathrm{~K}$ (above $45 \mathrm{~K}$ the $\mathrm{E}_{31 \mathrm{~h}}$ signal became difficult to resolve from the GaAs band-gap PL). A representative spectrum, taken at a bias of $0.5 \mathrm{~V}$ and $T=45$ $\mathrm{K}$ is shown in Fig. 2(b). At a lattice temperature of $45 \mathrm{~K}$, the thermal distribution of holes ( $4 \mathrm{meV}$ ) will probe electron energics up to $12 \mathrm{meV}$ from the subband minima. Since our experiments revealed no discernible broadening nor any changes in relative intensity of the PL peaks, we conclude that the $5 \mathrm{~K}$ measurements probe the entire $\mathrm{E} 3$ and $\mathrm{E} 4$ distributions, and that Eq. (1) is valid for the calculation of the $n_{4} / n_{3}$ ratio.

The oscillator strengths were calculated from solutions to the Schrodinger equation for the QW potential in the presence of an electric field. Since the states are not true bound states, but tunneling resonances, plane wave boundary conditions were used on the low energy side of the structure. Electric field values were obtained by comparing the bias dependence of the $E_{11 \mathrm{~h}}$ peak position with the calculated clectric field dependence of the $E_{1 \mathrm{~h} h}$ energy due to the quantum confined Stark effect. ${ }^{8}$ In general, the oscillator strengths will depend on in-plane wave vector for $k_{x y}>0 .{ }^{9}$ However, we have calculated, using the HHI dispersion relation for our potential, that there is little change in either $f_{3}$ or $f_{4}$ for $k_{x y}$ up to about $4 \%$ of the Brillouin zone boundary wave vector. For instance, at a bias of $0.5 \mathrm{~V}$ the $f_{4} / f_{3}$ ratio at $k_{x y}=1.5 \times 10^{6} \mathrm{~cm}^{-1}$ (the maximum wave vector probed by HH1 holes at $45 \mathrm{~K}$ ) is calculated to be only $10 \%$ greater than the value at $k_{x y}=0$, For the range of $k$ space probed by the $10 \mathrm{~K}$ hole population $\left(k_{x y} \leqslant 7.5 \times 10^{5} \mathrm{~cm}^{-1}\right)$, the variations are even smaller, and are therefore ignored in our calculations.

The $f_{4} / f_{3}$ ratio was calculated in this way throughout the bias range of the $E 4$ resonance, increasing from a value of $0.21+0.04$ at $0.44 \mathrm{~V}$ to $0.28 \pm 0.05$ at the peak of the resonance. The uncertainty in $f_{4} / f_{3}$ is due to estimated uncertainties in material parameters and in the determination of the electric field. Band bending due to charge buildup in the QW was ignored in the calculations of $f_{4} / f_{3}$. The majority of the charge buildup occurs in the E1 level, the E1 population being of the order of $10^{4}$ times greater than those of the excited states. Inclusion of band bending arising from an electron density in $\mathrm{E} 1$ of $10^{11} \mathrm{~cm}^{-2}$ on resonance ${ }^{10}$ leads to a decrease in the $f_{4} / f_{3}$ values (and a corresponding increase in $\left.n_{4} / n_{3}\right)$ of $\sim 20 \%$.

The values of $n_{4} / n_{3}$ obtained from Eq. (1) are plotted in Fig. 1(e). It is apparent that $n_{4}>n_{3}$ throughout the bias range of the $\mathrm{E} 4$ resonance, suggesting that a population inversion is 


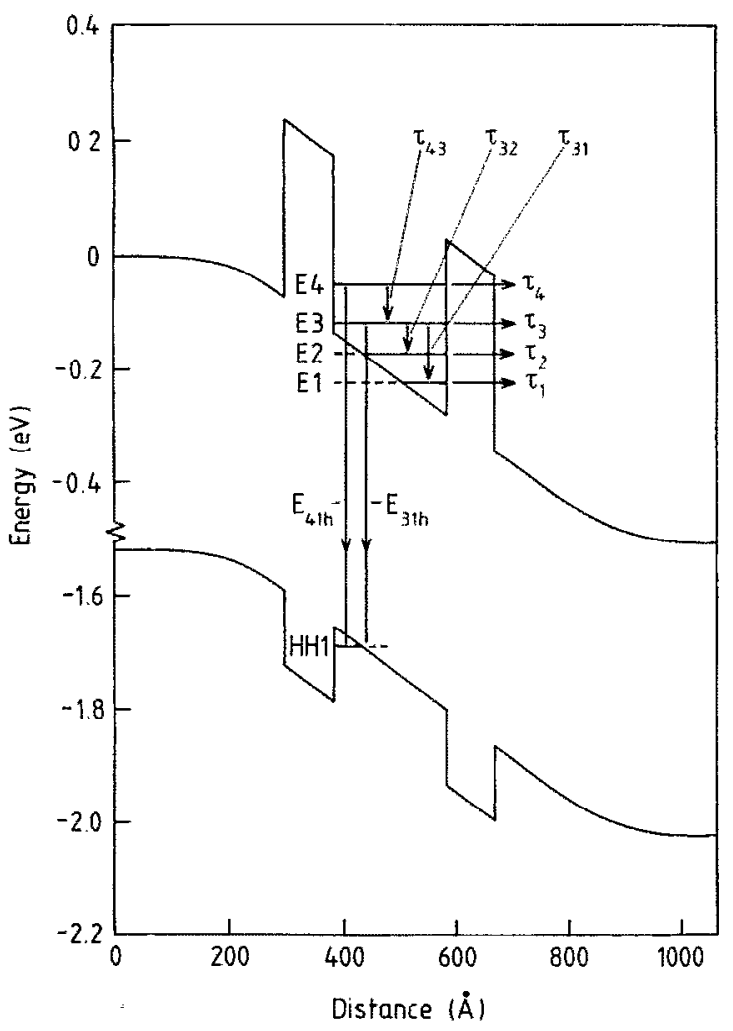

FIG. 3. Schematic band-edge diagram of the DBRTS at a bias of $0.5 \mathrm{~V}$. The tunneling-out times $\left(\tau_{1}-\tau_{4}\right)$ from the QW levels are indicated, as are the relcvant intersubband scattcring timcs, $\tau_{43}, \tau_{32}$, and $\tau_{31}$,

obtained between E4 and E3 when electrons tunnel into E4. We now show that such a population inversion is consistent with a rate analysis of the populations of E3 and E4 when the structure is biased at the $\mathrm{E} 4$ resonance.

As indicated in Fig. 3, when electrons tunnel into E4 they can subsequently either tunnel directly out of the QW, or scatter down to lower QW levels before tunneling out. With the DBRTS biased at the E4 resonance, the E3 population obeys the following rate equation: ${ }^{11}$

$$
\frac{d n_{3}}{d t}=\frac{n_{4}}{\tau_{13}}-\frac{n_{3}}{\tau_{3}}-\frac{n_{3}}{\tau_{32}}-\frac{n_{3}}{\tau_{31}}-\frac{n_{3}}{\tau_{R 3}},
$$

where $\tau_{43}, \tau_{32}$, and $\tau_{31}$ are the scattering times from $\mathrm{E} 4$ to $E 3, E 3$ to $E 2$, and from $E 3$ to $E 1$ respectively, $\tau_{3}$ is the tunneling-out time from $\mathrm{E} 3$, and $\tau_{R 3}$ is the characteristic time for recombination with $\mathrm{HH} 1$ holes. The intersubband scattering time for subband spacings greater than the LO phonon energy ( $\hbar \omega_{\mathrm{LO}}=36 \mathrm{meV}$ ) is $\sim 0.5 \mathrm{ps},{ }^{12}$ whereas $\tau_{3}$ is calculated to be $\sim 250 \mathrm{ps}$ at a bias of $0.5 \mathrm{~V}$, and $\tau_{R 3}$ will very likely be greater than $10 \mathrm{~ns}^{7}$ Thus, the tunneling-out and recombination terms may be neglected in comparison with the intersubband scattering terms in Eq. (2), to give, in the steady state

$$
n_{4} / n_{3}=\left(\tau_{43} / \tau_{32}\right)+\left(\tau_{43} / \tau_{31}\right)
$$

LO phonon scattering of electrons between parallel, parabolic subbands separated by energy $\Delta E$ requires the participation of a phonon of wave vector $q \propto\left(\Delta E-\hbar \omega_{\mathrm{LO}}\right)^{1 / 2}$. Since the strength of the Frohlich interaction between electrons and LO phonons decreases with increasing $q,{ }^{12}$ the scattering time is expected to increase with $\Delta E$. Thus, $\tau_{43}(\Delta E=67 \mathrm{meV})>\tau_{32}(\Delta E=47 \mathrm{meV})$ giving, from Eq. (3), $\left(n_{4} / n_{3}\right)>1$, consistent with the observed population inversion. ${ }^{13}$ Using the model of Ref. 12 we have calculated values for the scattering times in Eq. (3), using appropriate form factors for the transitions involved, and obtain $n_{4} / n_{3} \sim 2.3$, in good agreement with our measurements.

In conclusion, we have studied PL from the E3 and E4 excited states of the QW of a DBRTS under bias. Analysis of the PL has shown that the relative intensities of the $E_{31 h}$ and $E_{4 \mathrm{hh}}$ signals correspond to a higher population of electrons in E4 than in E3 when the structure is biased at the E4 resonance. We have shown that such a population inversion is consistent with the results of a rate analysis of the relative populations of $\mathrm{E} 3$ and $\mathrm{E} 4$ in this bias range.

This work was supported by the SERC, UK. We thank K. J. Nash and L. Eaves for very helpful discussions.

\footnotetext{
'R. F. Kazarinov and R. A. Suris, Fiz. Tekh. Poluprovodn. 6, 148 (1972) [Sov. Phys. Semicond. 6,120 (1972)].

${ }^{2}$ M. Helm, P. England, E. Colas, F. DeRosa, and S. J. Allen, Jr., Phys, Rev. Lett. 6374 (1989).

${ }^{3}$ A. Kastalsky, V. J. Goldman, and J. H. Abeles, Appl. Phys. Lett. 59, 2636 (1991).

${ }^{4}$ A. A. Andronov, Semicond. Sci. Technol. 7, B269 (1992).

${ }^{5}$ M. D. Sturge, Phys. Rev. 127, 768 (1962).

${ }^{6}$ H.-I. Polland, W. W. Rühle, J. Kuhl, K. Ploog, K. Fujiwara, and T. Nakayama, Phys. Rev. B 35, 8273 (1987).

${ }^{7}$ T. Matsusue and H. Sakaki, Appl. Phys. Lett. 50, I429 (1987); due to the very long tunneling-out time from $\mathrm{HH}$, the lifetime is determined by recombination with electrons in the QW.

${ }^{8}$ D. A. B. Miller, D. S. Chemla, T. C. Damen, A. C. Gossard, W. Wiegmann, T. H. Wood, and C. A. Burrus, Phys. Rev. B 32, 1043 (1985).

${ }^{\text {9 } Y . ~ C . ~ C h a n g ~ a n d ~ J . ~ N . ~ S c h u l m a n, ~ i b i d . ~ 31, ~} 2069$ (1985).

${ }^{10}$ M. S. Skolnick, D. G. Hayes, P. E. Simmonds, A. W. Higgs, G. W. Smith, H. J. Hutchinson, C. R. Whitehouse, L. Eaves, M. Henini, O. H. Hughes, M. L. Leadbeater, and D. P. Halliday, Phys. Rev. B 41, 10754 (1990).

${ }^{11}$ Since the $I-V$ peak to valley ratio at the $\mathrm{E} 3$ resonance is $5.5: 1$, we ignore filling of $\mathrm{E} 3$ by nonresonant processes.

${ }^{12}$ R. Ferreira and G. Bastard, Phys. Rev. B 40, 1074 (1989).

${ }^{13}$ For a similar three level system, where instead the level separations are less than the LO phonon energy, Allen et al. [S. J. Allen, G. Brozac, E. Colas, F. DeRosa, P. England, J. Harbinson, M. Helm, L. Florez, and M. Leadbeater, Semicond. Sci. Technol. 7, B1 (1992)] have shown that a population inversion is not observed, since the acoustic phonon scattering rate increases with increasing level separation (Ref. 12).
} 